10. Professor Dickson has found certain polynomial modular invariants and properties of such invariants for the case of a binary $n$-ic and a prime modulus $P$. In this paper Professor McAtee generalizes these invariants for the case of a binary quadratic and modulus a power of a prime, $P^{\lambda}$. Then these invariants are specialized for the case $P=\lambda=2$ and a fundamental system is exhibited modulo 4 .

11. With a view toward applications to the expansion of functions in terms of given functions, Professors Hedrick and Ingold have been led to study the properties of a general linear distributive operation $L[f(x), \varphi(x)]$ on a pair of functions $f(x)$ and $\varphi(x)$. In this paper, it is shown that such an operation leads at once to a general formula of which Bessel's inequality is a special case. Other related formulas are also generalized.

A similar operator, defined only for the product $f(x) \cdot \varphi(x)$ has been studied by Moore. (See Bulletrn, volume 18, pages 334-362.)

12. Professor Roever gives a geometric explanation of elliptical light curves seen in a highly scratched plate illuminated by a point source.

O. D. KellogG, Secretary of the Sectiou.

\title{
NOTE ON CONJUGATE NETS WITH EQUAL POINT INVARIANTS.
}

BY DR. G. M. GREEN.

(Read before the American Mathematical Society, September 4, 1917.)

IN my second memoir on conjugate nets on a curved surface, ${ }^{*}$ I gave a new characterization of conjugate nets with. equal Laplace-Darboux invariants. The theorem as there stated, however, is not quite complete, and it is the purpose of this note to supply the necessary refinement, as well as to generalize the theorem and put it into relation with another,

* Amer. Journal of Mathematics, vol. 38 (1916), pp. 287-324. See in particular p. 313. 
which is involved in a different characterization of conjugate nets with equal invariants.

Using the notation of the paper just cited, let

$$
y^{(k)}=y^{(k)}(u, v) \quad(k=1,2,3,4)
$$

be the point equations, in homogeneous coordinates, of a surface $S$ referred to a conjugate net. The four functions $y$ are a fundamental system of solutions of the completely integrable system of partial differential equations

$$
\begin{aligned}
& y_{u u}=a y_{v v}+b y_{u}+c y_{v}+d y, \\
& y_{u v}=b^{\prime} y_{u}+c^{\prime} y_{v}+d^{\prime} y .
\end{aligned}
$$

The minus first and first Laplace transforms of the point $y$ of the surface are respectively

$$
\rho=y_{u}-b^{\prime} y, \quad \sigma=y_{v}-a^{\prime} y .
$$

The line joining these two points we shall call with Wilczynski the ray of the point $y$, and the congruence of rays the ray congruence. The developables of the ray congruence correspond to a net of curves on $S$, which we shall call the ray curves, and whose differential equation is*

$$
a H d u^{2}-\mathfrak{D} d u d v-K d v^{2}=0,
$$

where

$$
H=d^{\prime}+b^{\prime} c^{\prime}-b_{u}{ }^{\prime}, \quad K=d^{\prime}+b^{\prime} c^{\prime}-c_{v}{ }^{\prime}
$$

are the Laplace-Darboux invariants, and

$$
\mathfrak{D}=d+a{b^{\prime}}^{2}-{c^{\prime}}^{2}+a b_{v}{ }^{\prime}-c_{u}{ }^{\prime}+b^{\prime} c+b c^{\prime} .
$$

Two ray curves pass through each point $y$ of the surface, and we shall call their tangents at the point $y$ the ray tangents of $y$. The ray tangents are the lines which join $y$ to the points $y_{u}+(d v / d u)_{1} y_{v}, y_{u}+(d v / d u)_{2} y_{v}$, where $(d v / d u)_{1}$ and $(d v / d u)_{2}$ are the roots of equation (2) considered as a quadratic in $d v / d u$.

The focal points of the ray $\rho \sigma$ are given by the formulas $\dagger$

$$
R=\rho+r_{1} \sigma, \quad S=\rho+r_{2} \sigma,
$$

where $r_{1}$ and $r_{2}$ are the roots of the quadratic

$$
H r^{2}+\mathfrak{D} r-a K=0 .
$$

* G. M. Green, loc. cit., p. 309.

$\dagger$ Loc. cit., p. 309. 
A comparison of equations (2) and (3) will show that the ray tangents meet the ray in the focal points of the ray if and only if the conjugate net has equal Laplace-Darboux invariants, provided, however, that $\mathfrak{D}$ is not zero.* If $\mathfrak{D}$ is zero, the quadratics (2) and (3) would coincide not only if $H=K$, but also if $H=-K$. If, however, $H=-K$, and $\mathfrak{D}$ is zero, equation (2) becomes

$$
H\left(a d u^{2}+d v^{2}\right)=0 .
$$

If, then, $H$ is different from zero (if it were zero, we should have again $H=K$ ) the differential equation of the ray curves becomes

$$
a d u^{2}+d v^{2}=0,
$$

which is the differential equation of the asymptotics. In other words, if the ray curves coincide with the asymptotic curves, then the ray tangents will meet the ray in the focal points of the ray.

However, the geometric facts enable us to state the entire situation more sharply. An analytic proof of these factswe shall presently supply a geometric proof-will show that in the first case, i. e., $H=K$, the tangent to one of the ray curves through $y$ meets the ray in the focal point which corresponds to the other ray curve, whereas in the second case, i. e., $\mathfrak{D}=0$ and $H=-K$, the tangent to a ray curve meets the ray in the focal point belonging to that ray curve. So we may state our characterization of conjugate nets with equal invariants as follows:

A necessary and sufficient condition that a conjugate net have equal point invariants is that for every point of the surface the tangents to the two ray curves at the point meet the corresponding ray in the focal points of the ray, the tangent to either ray curve meeting the ray in the focal point which corresponds to the other ray curve. If each ray tangent meets the ray in the focal point which corresponds to it, the ray curves coincide with the asymptotics of the surface. In this case $H=-K$ and $\mathfrak{D}=0$. If $H=K=\mathfrak{D}=0$, the ray curves are indeterminate.

In this connection it will be well to recall Wilczynski's theorem, $\dagger$ that a necessary and sufficient condition that a con-

* Loc. cit., p. 313, except for the proviso at the end of the present statement.

$\dagger$ E. J. Wilczynski, "The general theory of congruences," Transactions Amer. Math. Society, vol. 16 (1915), pp. 311-327. 
jugate net have equal point invariants is that its ray curves form a conjugate net. Using this theorem, we may prove the previous one on the basis of the following very general theorem:

Let $t_{1}$ and $t_{2}$ be the ray tangents of a point of the surface, and $\Phi_{1}, \Phi_{2}$ the corresponding focal points, respectively, of the ray. Then the ray is met in $\Phi_{1}$ by the tangent to the surface which is conjugate to $t_{1}$, and in $\Phi_{2}$ by the tangent conjugate to $t_{2}$.

We shall give a geometric proof of this theorem; the proof will show that a perfectly good theorem may sometimes be obtained by merely dualizing a trivial statement. Let us regard the surface $S$ as the locus of points $y$, and the envelope of the corresponding tangent planes $\Pi$. By polar reciprocation, $S$ goes into a surface $S_{1}$, the point $y$ becoming the tangent plane $\Pi_{1}$, and the plane $\Pi$ becoming the point of contact $y_{1}$ on $S_{1}$. The points $\rho$ and $\sigma$ become the osculating planes of the parametric curves $u=$ const., $v=$ const., respectively, at the point $y_{1}$ on $S_{1}$. These osculating planes meet in a line, which Wilczynski has called the axis of the point $y_{1}$. The totality of axes constitute the axis congruence, and the curves on $S_{1}$ which correspond to the developables of this congruence are called the axis curves. The points of a curve $C$ on $S$, i. e., a one-para neter farnily of points, correspond to a one-parameter family of planes tangent to $S_{1}$. The points of contact of these planes constitute a curve $C_{1}$ on $S_{1}$, which we may consider as the curve corresponding to $C$. But the tangent to $C$ at $y$ does not go over into the tangent to $C_{1}$ at $y_{1}$, but into the conjugate direction. The focal points of the ray $\rho \sigma$ correspond to the focal planes* of the axis congruence of $S_{1}$. An axis tangent on $S_{1}$, i. e., a tangent to an axis curve, corresponds to the direction on $S$ which is conjugate to a ray tangent on $S$. If we recall that a focal plane of a line of a congruence is tangent to a developable of the congruence all along a line of the congruence, and if we observe that the developables of the axis congruence actually cut the surface $S_{1}$ in the axis curves, then it becomes evident that the axis tangents lie in the corresponding focal planes. If we dualize this situation on $S_{1}$, we obtain the situation on $S$ which is described in the theorem which we set out to prove.

Having, then, shown that the direction conjugate to one ray tangent meets the ray in that focal point of the ray which

* The focal planes of an axis are the planes tangent, along the axis, to the two developables of the axis congruence to which that axis belongs. 
corresponds to the other ray tangent, and recalling Wilczynski's theorem, we are led at once to the final form in which we stated the geometric characterization of conjugate nets with equal point invariants. This characterization may of course be established analytically, and independently of Wilczynski's theorem, so that, on the basis of our general theorem, Wilczynski's characterization may then be obtained from ours.

HARVARD UNIVERSITY, August 22, 1917.

\section{ON THE DIFFERENTIABILITY OF ASYMPTOTIC SERIES.}

BY DR. J. F. RITT.

(Read before the American Mathematical Society, October 27, 1917.)

THE question of the differentiability of asymptotic series seems not to have received adequate treatment.* Writers on the theory of asymptotic convergence content themselves always with stating that if $P(x)$ has the asymptotic representation

$$
P(x) \sim a_{0}+a_{1} x+a_{2} x^{2}+\cdots+a_{n} x^{n}+\cdots,
$$

it may not have a derivative at all, and that even if a derivative does exist, the derivative may not admit of asymptotic development.

A failure to distinguish between the real and complex domains, in this connection, is responsible for a serious lacuna, which it is the purpose of this note to fill.

Let $P(x)$, defined in a sector with vertex at the origin, be analytic within the sector, in the neighborhood of the origin, and continuous on the sides of the sector, and at the origin. Let $P(x)$ have the asymptotic development of finite order

$$
P(x)=a_{0}+a_{1} x+a_{2} x^{2}+\cdots+a_{n} x^{n}+\epsilon(x) x^{n},
$$

where $n \geqq 2$ and where $\epsilon(x)$ goes to zero with $x$. We say that

* Since writing this note, I have ascertained by correspondence with Professor Birkhoff that he was familiar with and had used the result given here, but failed to publish it, being under the impression that it was contained in an article by W. B. Ford (Bull. de la Soc. Math. de France, 1911, p. 347). 